\title{
PEAPOD limits developmental plasticity in Arabidopsis.
}

Derek W.R. White ${ }^{* 1,2}$

1 Institute of Fundamental Sciences, Massey University, 4442 Palmerston North, New Zealand, ${ }^{2}$ AgResearch, Private Bag 11008, Palmerston North, New Zealand.

*Correspondence and requests for materials should be addressed to d.w.white@massey.ac.nz). ${ }^{1}$ Present address.

Short title: PPD limits developmental plasticity.

\section{Abstract}

Higher plants utilise developmental plasticity to adapt to changes in the environment, especially to variations in light. Much of this change in growth and development involves the light-mediated regulation of multiple hormone pathways. However, despite considerable progress towards understanding the molecular processes controlling light signalling and hormone activity, regulatory mechanisms preventing exaggerated plant developmental responses are not well understood. Here I report that the $P P D$ regulatory complex has a crucial role in limiting developmental plasticity in Arabidopsis. Reductions in PPD or KIX8/9 gene expression resulted in; tolerance to ABA inhibition of seed germination, hypocotyl elongation, increases in stomata on hypocotyls, cambial cell proliferation and seed weight, and delayed flowering. Transcript profiling and analyses of hormone responses and genetic interactions established PPD modulates developmental plasticity, mainly by a combination of transcriptional activation and repression of genes controlling CRY/PHY light signalling and ABA, auxin, brassinosteroid, cytokinin and gibberellin homeostasis.

\section{Introduction}

Because they are sessile, higher plants have evolved extensive developmental plasticity to acclimatize to changes in the environment. This capacity to alter plant morphology involves coordinated changes in cell proliferation and expansion during growth and development, throughout the life cycle. Light is one of the main environmental signals influencing plant developmental plasticity. In addition to its importance for photosynthesis, light also affects many aspects of plant development including seed germination, seedling de-etiolation, photomorphogenesis, stem elongation, leaf size, stomatal density, shade avoidance, flowering time, and seed development ${ }^{1-3}$. The perception of light by photoreceptors can cause changes in the transcription of $\sim 30 \%$ of genes in the Arabidopsis genome, including 
transcription factors that modulate hormonal, metabolic, stress, pigment biosynthesis, and defence pathways ${ }^{4-5}$.

Plants perceive light using multiple photoreceptors: in Arabidopsis there are five red/far red absorbing phytochromes (phyA - phyE), two blue absorbing cryptochromes (cry1 and cry2), two blue/UV absorbing phototropins (phot1 and phot2), and a UV-B receptor, UVR8 ${ }^{6-7}$. Although individual photoreceptors elicit specific developmental responses, light signals mediated by different photoreceptors are also integrated and can have similar effects on transcription and morphology during seedling photomorphogenesis. The integration point for multiple light receptors appears to be a few light-induced transcription factors first identified as positive regulators of seedling photomorphogenesis, one of which is the bZIP transcription factor, $\mathrm{HY}^{8-11}$. HY5 acts downstream of the phytochrome, cryptochrome, and UV-B photoreceptors, directly regulating expression of $\sim 3,800$ genes in the Arabidopsis genome including genes involved in photosynthesis, light signalling, hormone biosynthesis and catabolism, circadian rhythm, flavonoid accumulation, and nutrient assimilation ${ }^{9}$. In the dark or shade a negative regulator of photomorphogenesis, COP1, a RING-finger type ubiquitin E3 ligase, physically interacts with HY5 and mediates its degradation ${ }^{12}$. HY5 degradation is enhanced by the physical interaction of COP1 with SPA proteins ${ }^{12-13}$. When seedlings are exposed to light, activated cry1, cry2, phyA, or phyB directly interact with SPA proteins resulting in, dissociation of the COP1-SPA complex, accumulation of HY5, and promotion of photomorphogenic development ${ }^{14-17}$. This interaction of light, photoreceptors and both negative and positive regulators of light signalling provides a mechanism to fine tune plant growth and developmental responses to changes in light quantity and quality.

Coordinated changes in the homeostasis and signalling of multiple hormones are an essential component of light-mediated alterations in growth and development ${ }^{3,18}$. Hormones such as auxin, brassinosteroid (BR), and gibberellin (GA) antagonise photomorphogenesis, whereas it is promoted by abscisic acid (ABA). For example, light influences auxin levels by coordinated regulation of the transcription of TAA1, an auxin biosynthesis gene, and SUR2, a cytochrome P450 monooxygenase that alters auxin levels by converting indole acetic acid to indole glucosinolates ${ }^{18-21}$. Active phyB reduces auxin levels by the up-regulation of SUR2, and down-regulation of TAA1 transcription, whereas inactivation of phyB results in elevated levels of auxin due to reduced SUR2 and increased TAA1 transcription. Similarly, the lightmediated regulation of $B R, G A$, and $A B A$ levels are modulated by a combination of metabolic processes; biosynthesis, conjugation, and catabolism ${ }^{22-34}$. However, despite extensive progress made in recent years towards understanding the mechanisms controlling light signalling and hormone activity, one of the fundamental questions in plant biology 
remains how plants coordinate and optimise growth and development in a constantly changing environment.

Higher plants are able to adapt to changes in the environment by altering the size of their leaves. In Arabidopsis, as the leaf grows cells differentiate and undergo cell expansion, starting at the tip of the leaf and progressing to the base ${ }^{35}$. At the same time meristemoids, stomatal lineage precursors, undergo a limited number of asymmetric cell divisions before forming stomatal guard cells and associated pavement cells ${ }^{36}$. Meristemoid cell proliferation is an important determinant of leaf size in Arabidopsis, because meristemoid cells generate a large portion of the epidermal pavement cells (48\% in leaves and $67 \%$ in cotyledons) ${ }^{37}$. To date the only mechanism reported specifically limiting meristemoid cell proliferation involves PPD1 and PPD2, and interacting protein partners ${ }^{38-39}$. Deletion or silencing of the tandemly repeated PPD genes in Arabidopsis extends division of meristemoids in the epidermis, increasing stomatal density and leaf size, whereas overexpression of PPD1 results in the opposite phenotype. The PPD proteins belong to class II of the plant-specific TIFY family of transcription regulators. This group of proteins, which includes the JAZ proteins, negative regulators of jasmonic acid signalling, have a central ZIM domain that mediates interactions between TIFY proteins and also with the EAR domain containing NINJA, which acts as an adaptor for the corepressor TPL ${ }^{40-41}$. The PPD proteins are distinguished from other TIFY proteins by a unique $\mathrm{N}$-terminal PPD domain. This domain mediates the interaction of PPD proteins with the EAR domain containing TPL adaptor proteins $\mathrm{KIX} 8$ and $\mathrm{KIX9}{ }^{39}$. Plants with mutant kix8 and kix9 have a similar leaf phenotype to the $\Delta p p d$ deletion mutant. PPD proteins also physically interact with SAP, an F-box protein that forms part of a SKP1/Cullin/F-box E3 ubiquitin ligase complex which targets the PPD proteins for degradation $^{42}$. Plants overexpressing $S A P$ also have a leaf phenotype similar to the $\triangle p p d$ mutant. Hence the PPD proteins and their interacting partners provide a molecular mechanism to change leaf size.

To assess if the PPD genes have a more extensive role in the control of plant developmental plasticity I first characterised the phenotypes of Arabidopsis $\Delta p p d$ deletion mutant and PPD1 overexpression genotypes throughout the life cycle. I then used genomewide transcript profiling to identify genes with altered transcription levels due to PPD deletion. Hormone and hormone biosynthesis inhibitor treatments, and genetic analysis were used to confirm alterations in hormone homeostasis detected by transcript profiling. Finally, I used a combination of genetic and gene expression analyses to provide insights into how PPD proteins regulate both hormone homeostasis and light signalling. Here I report an extensive role for the $P P D$ genes as master regulators modulating the plasticity of many aspects of development. 


\section{Results}

\section{The PPD regulatory complex controls diverse aspects of growth and development.}

To further understand the role that PPD genes have in the control of plant development a comparison was made of cell proliferation and growth characteristics in Arabidopsis wildtype, ppd deletion mutant $(\triangle p p d)$, and $\triangle p p d:: P P D 1$ overexpressor (PPDOE) genotypes. As previously reported $\Delta p p d$ mutant plants had enlarged dome shaped leaves with elongated petioles and larger cotyledons than wild type, whereas overexpression of PPD1 resulted in plants with smaller leaves and cotyledons, and shorter petioles ${ }^{38}$ (Fig 1a). Seedlings of the $\triangle p p d$ mutant had increases in hypocotyl length and stomata numbers on the hypocotyl (Fig. 1a,b,e,f). Surprisingly, PPDOE seedlings also had an increase in hypocotyl length. However, stomata numbers on the hypocotyls of PPDOE were less than wild type. Additionally, PPDOE primary roots were shorter than wild type (Supplementary Fig. 1).

I previously reported that $\triangle p p d$ has a more extensive, and PPDOE a reduced, vascular network in cotyledons ${ }^{38}$. Histochemical staining of the roots of PPD1promoter-GUS plants indicated PPD1 is expressed within the vascular stele (Supplementary Fig. 2). Furthermore, up-regulation of CYCB1;1-GUS, a marker of cell-cycle progression, in both the root and leaf vascular of $\triangle p p d$, suggested a role for $P P D$ genes controlling (pro)cambium cell proliferation (Supplementary Fig. 3). This function was confirmed with the observation that $\Delta p p d$ had an increase, and PPDOE a reduction, in procambium cell number in cotyledon vascular traces (Fig. 1c,g). Similarly, cambium cell proliferation increased in $\Delta p p d$, and decreased in $P P D O E$, mature inflorescence stems (Supplementary Figs. 4 and 5). Furthermore, in a comparison of vascular development in seedling hypocotyls $\Delta p p d$ had increased xylem vessel number, whereas in PPDOE the amount of xylem was reduced (Supplementary Fig. $5)$.

I also observed that $\triangle p p d$ plants grown in a long-day photoperiod were delayed in flowering (Fig. 1h), whereas PPDOE flowered at the same time as wild type. In addition, seed of $\triangle p p d$ appeared to be larger than wild type, while seed of the PPDOE line were smaller (Fig. 1d). These seed size phenotypes were reflected in significant differences in seed weight, with a $53 \%$ increase for $\triangle p p d$ and a $22 \%$ decrease for PPDOE (Fig. 1i).

The role of the PPD genes in this multifaceted $\triangle p p d$ mutant phenotype was verified in two ways. Firstly, a PPD1 transgene was sufficient to complement the $\triangle p p d$ deletion phenotype (Figs.S5-6). For all the characters examined; hypocotyl length, stomata number per hypocotyl, cotyledon procambium cell number, stem cambium cell proliferation, hypocotyl xylem vessel number, flowering time and seed weight, the $\triangle p p d:: P P D 1$ line was not significantly different from wild type. Secondly, other genotypes with disruption of the PPD 
complex had phenotypes similar to the $\triangle p p d$ mutant. A transgenic line with partial silencing of PPD1 and PPD2 expression, ami-ppd, due to overexpression of an artificial microRNA ${ }^{39}$, had increased stomata numbers on the hypocotyl, delayed flowering time, and an increase in seed weight, similar to $\Delta p p d$ (Fig. 1f,h,i), and increases in hypocotyl length and procambium cell proliferation significantly greater than wild type (Fig. 1e,i). Also, in a pattern similar to $\Delta p p d$ and ami-ppd, kix8;kix9 mutants had significant increases in hypocotyl length, stomata numbers on the hypocotyl, flowering time, and seed weight (Fig. 1e,f,h,i). The complex phenotype resulting from altered PPD or KIX gene expression suggests the PPD transcriptional regulators have a role coordinating and controlling diverse aspects of growth and development, throughout the Arabidopsis life cycle.

\section{Genome-wide transcriptomics reveals PPD regulates light signalling and hormone}

metabolism. To obtain insight into transcriptional changes underlying the ppd deletion phenotype, RNA was isolated from whole seedlings of $\triangle p p d$ and wild type plants at 10 days post germination and compared by RNA-Seq genome-wide transcription analysis. A total of 2,830 genes were differentially expressed in $\Delta p p d$ compared to wild type, with 927 (33\%) up-regulated and 1,903 (67\%) down-regulated (Supplementary Table 1). Gene Ontology terms significantly over represented included those for; "response to light stimulus", "circadian rhythm", "hormone biosynthesis process", "regulation of hormone levels", and "stomatal complex development" (Fig. 2a). To determine which of these genes might be direct targets of the PPD proteins, the dataset was compared with published information on the genome binding sites of PPD2 ${ }^{39}$ (Supplementary Table 1). Significant enrichment of PPD2 targets was found among genes both up- and down regulated in the $\Delta p p d$ mutant (8.9\% or 251 , with $30 \%$ up-regulated) (Fig. 2d). By chance, PPD2 would be predicted to bind to $3.5 \%$ of genes in the dataset (1,191 targets out of 33,602 Arabidopsis genes). Consistent with the increased number of cells involved in stomatal development in the $\Delta p p d$ mutant, most of the genes known to be involved in regulation of meristemoid cell proliferation were present in the dataset, all up-regulated (Table IA). The initiation and proliferation of meristemoids is regulated by a basic helix-loop-helix transcription factor, $\mathrm{SPCH}^{43}$, which has a large number of binding sites in the genome $(8,327)^{44}$. These SPCH targets were crossreferenced with the $\Delta p p d$ differential gene expression dataset to determine the extent of targeting by SPCH (Supplementary Table 1). Significant enrichment of SPCH targets $(1,050)$ was found in the dataset (37\%), but with fewer up-regulated (12.5\%) than expected (Fig. 2d). By chance, SPCH would be expected to bind to $25 \%$ of genes in the dataset. There were fewer joint PPD + SPCH target genes in the dataset (26) than expected indicating limited functional overlap between the gene expression networks regulated by these two 
transcription factors. RT-qPCR expression analysis confirmed the up-regulation of SPCH, $T M M$, and ERL1 in $\triangle p p d$, while all three genes were down-regulated in PPDOE (Fig. 2b).

Other GO terms over represented in the dataset included those for "anthocyanin biosynthesis" and "light harvesting". Notably, genes with key roles in anthocyanin biosynthesis; CHS, CHI, FLS, and PAP1 were all down-regulated, as were light harvesting genes; CAB2, LHCA6, LHCB2.3, and LHC4.3. Since all of these genes are positively regulated by $\mathrm{HY}^{10}{ }^{10}$, putative $\mathrm{HY} 5$ binding sites ${ }^{9}$ were identified among genes in the $\triangle p p d$ dataset (Supplementary Table 1). HY5 was predicted to bind to 626 genes, a significant enrichment (22\%) compared to the by chance prediction of $11.6 \%$. About $21.6 \%$ of these HY5 targets in the dataset were up-regulated genes. There was also over representation of GO terms relating to pathogen defensive responses "defence response to fungus", and "defence response to bacterium" in the dataset, with most of the genes down-regulated.

Both the $\Delta p p d$ seedling phenotype and the over-abundance of "light signalling response" GO terms in the RNA-Seq dataset suggested PPD genes might act as positive regulators of photomorphogenesis. To explore this possibility the dataset was scanned for genes involved in light signalling, circadian rhythm and the photoperiodic control of flowering time. Selected examples are given in Table IB. The up-regulation of SPA1, a negative regulator of phyA signalling and photomorphogenesis ${ }^{12}$, and the down-regulation of $A F R$, a positive regulator of phyA signalling ${ }^{45}$, both putative PPD2 targets, is consistent with PPD proteins having a direct and high level role in the modulation of light signalling. Furthermore, the long hypocotyl phenotype and down-regulation of $P A R 1^{46}$ and $P I L 1^{47}$ genes in $\triangle p p d$ would be expected if the mutant was defective in light signalling. These changes in the expression of light signalling genes in the $\triangle p p d$ mutant were confirmed by RT-qPCR analysis, with the opposite pattern of expression in PPDOE (Fig. 2b).

Regulators of the circadian clock that interact with phyB, such as ELF3 and GI, and clock components PRR5, BOA, and CHE, were up-regulated (Table IB). However, these alterations in the transcription of circadian rhythm genes do not appear to explain the delayed flowering of $\triangle p p d$. Expression of the key flowering time gene $F T$, is mainly upregulated in long days by the transcriptional activator $\mathrm{CO}$, and significantly, expression of some of the multiple regulatory components determining the activity of $\mathrm{CO}$ have been altered in $\triangle p p d$. Examples include the up-regulation of TOE2 and SPA1, and the downregulation of $N F-Y B 2$, all putative targets of PPD2 (Table IB). Expression analysis confirmed the up-regulation of ELF3, TOE2 and BOA, and down-regulation of NF-YB2 in $\triangle p p d$ (Fig. 2b, Supplementary Fig. S9). 
The presence of genes involved in hormone metabolism in the $\Delta p p d$ differential expression dataset suggested a possible role for PPD in the regulation of BR, auxin, cytokinin (CK), GA and ABA activities (Table IC). As an example of changes to gene expression known to increase BR levels, a key biosynthesis gene, $D W F 4^{26}$, was upregulated, whereas genes involved in $B R$ inactivation, $B A S 1^{22}, U G T 73 C 5^{23}$, and $B A T 1^{32}$, or reduced biosynthesis, $J U B 1^{34}$, were down-regulated. Similarly, the auxin biosynthesis gene $T A A 1$, was up-regulated, whereas genes reducing auxin activity or response, SUR2, IAA14, IAA3, GH3.3, GH3.5, GH3.17, and genes for camalexin biosynthesis (CYP71A12, CYP79B2, CYP79B3), were all down-regulated. For CKs, a gene involved in activation, LOG5, was up-regulated, and a gene for degradation, CKX4, down-regulated. While the GA biosynthesis gene GA20ox3, and a GA responsive gene, GASA14, were both up-regulated, $R G A 1$, a negative regulator of GA response was also up-regulated. In contrast to these examples a gene responsible for the regeneration of $A B A$ from a pool of inactive $A B A$ glucosides, BG1, and genes involved in ABA responses: bZIP1, RD22, RD29A, KIN2, FIB, COR15a, COR413-PMI, were all down-regulated. Since $b Z I P 1$, a key positive regulator of responses to $A B A$ also activates genes involved in carbon and nitrogen metabolism ${ }^{48}$, the dataset was scanned for evidence of a reduction in expression of $\mathrm{C}$ - and $\mathrm{N}$ - metabolism genes. Interestingly, as would be expected for reduced bZIP1 activity, genes involved in Cmetabolism (BCA6, PPDK, ATBETAFRUCT4, BAM9, AS6, SWEET2, SWEET11, SWEET13, SWEET17, SUC2, SUC5) and N-metabolism (GDH2, TYROSINE AMINO TRANSFERASE, ASN1, IVD, PRODH1, BCE2, THA1, MCCA) were all down-regulated in $\triangle p p d$.

Differential expression in the mutant was confirmed for; DWF4, BAS1, BAT1, JUB1, TAA1, SUR2, IAA3, IAA14, GH3.3, GH3.5, GA200x3, GASA14, RGA1, BG1, bZIP1, FIB, RD29A, CKX4 and LOG5 (Fig. 2c, Supplementary Fig. S9). Where examined, expression in the PPDOE genotype was generally the opposite of the deletion mutant or similar to wild type, the notable exception being for GA20ox3, where expression was up-regulated in both $\triangle p p d$ and PPDOE. These patterns of gene expression in the $\triangle p p d$ mutant are consistent with enhanced activities for BR, GA, auxin and $C K$, and reduced sensitivity to ABA.

Although it has been reported that auxins, CKs and GAs regulate vascular (pro)cambium cell proliferation ${ }^{49}$, the dataset was also scanned for putative direct targets of PPD that might explain the $\triangle p p d$ and PPDOE cambial cell proliferation phenotypes. A possible candidate, $A C L 5$, was down-regulated. It has been reported that an ac/5 loss-of-function mutant has both increased vascular (pro)cambium cell proliferation and xylem cell differentiation ${ }^{50}$, characteristics similar to the $\triangle p p d$ mutant. Reduced expression of ACL5 in the $\triangle p p d$ mutant was confirmed by RT-qPCR expression analysis (Fig. 2b). 
Multiple hormone responses are modulated by PPD. To confirm PPD genes modulate hormone activity, wild type, $\triangle p p d$, and PPDOE genotypes were evaluated for responses to hormone and/or hormone biosynthesis inhibitor treatments (Fig. 3). Treatment with brassinazole (BRZ, an inhibitor of BR biosynthesis), reduced hypocotyl elongation in wild type seedlings grown in darkness, while treatment with the BR epibrassinolide (BL) reduced root growth in the light (Fig. 3a,b). The $\triangle p p d$ mutant was relatively insensitive to BRZ inhibition of hypocotyl elongation in the dark, but hyper-responsive to BL inhibition of root growth, while PPDOE had the opposite response. BRs also influence stomata development on the hypocotyl, with BL treatment increasing and BRZ decreasing the number of stomata (Fig. 3c). The $\triangle p p d$ mutant was hyper-responsive to $\mathrm{BL}$ stimulation and relatively insensitive to $\mathrm{BRZ}$ inhibition of stomata number, while in PPDOE there were reduced responses to both $\mathrm{BL}$ and the inhibitor. A similar set of experiments was used to determine responses to $\mathrm{GA}$ and the GA biosynthesis inhibitor paclobutrazol (PAC). Wild type seedlings responded to GA treatment with hypocotyl elongation and increased stomata number on the hypocotyl, whereas PAC treatment restricted both (Fig. 3d,e). Interestingly, hypocotyl elongation of both $\triangle p p d$ and PPDOE was hyper-responsive to GA and relatively insensitive to PAC, compared with wild type. Together with the up-regulation of a gene for GA biosynthesis in both genotypes, these responses suggest increased GA activity in PPDOE hypocotyls. However, the hyper-response to GA and insensitivity to PAC in stomata number on hypocotyls of $\triangle p p d$, together with the relative insensitivity of PPDOE to either treatment, suggests PPD's may also limit responses to GA (Fig. 3e). To assess auxin response wild type and $\Delta p p d$ seeds where germinated in the presence of $5 \mu \mathrm{M}$ or $50 \mu \mathrm{M}$ picloram (a synthetic auxin). Low concentrations of picloram stimulated and high concentrations inhibited hypocotyl elongation of the wild type. The increased sensitivity of $\Delta p p d$ to picloram observed is consistent with the mutant having an increase in endogenous auxin activity (Fig. 3f). Similarly, the hypersensitivity of $\triangle p p d$ to root growth inhibition by the synthetic cytokinin, 6-benzyl aminopurine (BAP), and the reduced response of PPDOE, suggests PPD's also modulate endogenous cytokinin activity (Fig. 3g). 
As the gene expression profile in $\triangle p p d$ suggested a possible role for PPD's in the modulation of ABA responses, genotypes with altered $P P D$ complex expression were tested for sensitivity to ABA inhibition of seed germination (Fig. 3h,i). The insensitivity to ABA inhibition of seed germination of the $\triangle p p d$, ami-ppd and kix8;kix9 genotypes and the wild type like response of PPDOE, is consistent with PPD's acting as positive regulators of ABA response.

PPD regulates $B R$ biosynthesis. Transgenic plants with overexpression of either the BR biosynthesis gene DWF4 (DWF4ox) $)^{51}$ or the BR receptor BRI1 (BRI1ox) ${ }^{52}$ have phenotypes similar to $\triangle p p d$, with elongated hypocotyls, increased stomata numbers on hypocotyls, ABA insensitive germination and increased seed weights (Fig. 4a-d). In genetic interaction analyses PPDOE was epistatic to DWF4ox or BRI1ox. Furthermore, when $\triangle p p d$ was combined with a partial loss-of-function mutant of $D E T 2^{53}$, a BR biosynthesis gene functioning upstream of DWF4, the double mutant (det2;ppd) had some increase in hypocotyl elongation, stomata numbers on hypocotyls, and insensitivity to ABA inhibition of seed germination, compared to det2. However, det2 was epistatic to $\Delta p p d$ for both seed weight and the inhibition of hypocotyl elongation in dark grown seedlings (Fig. 4d-f). Collectively, these results are consistent with PPD's acting as negative regulators of BR biosynthesis. RT-qPCR analysis indicated that there was no change in the expression of other genes involved in BR biosynthesis (CPD, BR6ox2), or signalling (BIN2) or transcriptional activation (BES1, BZR1, BIM2, BEH2, BEH3) in $\triangle p p d$ or PPDOE (Supplementary Fig. S9).

Interactions between $P P D, C R Y 1$ and $P H Y B$, modulate light signalling. Because transcriptomic analysis indicated PPD's may regulate some of the initial steps in the light signalling cascade by repressing SPA1 and activating AFR transcription, genetic interactions between $P P D$ and $C R Y 1$ or $P H Y B$ were evaluated. Seedlings of a cry1 loss-of-function mutant had elevated stomata numbers on elongated hypocotyls and increased procambium cell proliferation in vascular tissues (Fig. 5b-d). Genetic interaction in the double cry1;ppd mutant was additive for hypocotyl length and stomata number on hypocotyls, but not for procambium cell proliferation where cry $1, \triangle p p d$ and the double mutant were all similar. For all these characteristics PPDOE was epistatic to cry1, suggesting that the reduced SPA1 and/or elevated AFR expression in PPDOE (Fig. 2b) counteracted the defect in CRY1 signalling. Seedlings of a phyB loss-of-function mutant also had increased stomata numbers on hypocotyls (Supplementary Fig. S7). Plants with overexpression of phyB (phyBOE), exhibited short hypocotyls, a reduction in stomata number on hypocotyls, early flowering, insensitivity to ABA inhibition of seed germination (Fig. 5), and reduced root growth (Supplementary Fig. S1). Procambium cell proliferation and seed weight of phyBOE was 
similar to wild type. Overexpression of phyB was epistatic to $\Delta p p d$ for most of the parameters examined; hypocotyl length, stomata numbers on hypocotyls, procambium cell proliferation, flowering time, seed weight, and root growth. However, leaf curvature, and the extended proliferation of meristemoids during leaf development where similar to $\Delta p p d$ (Fig $5 a$, Supplementary Fig.8). While the number of stomata on hypocotyls at $5 \mathrm{dpg}$ was similar to phyBOE there was also extensive additional meristemoid cell proliferation on the hypocotyls of phyBOE;ppd seedlings. Hence, phyB overexpression counteracted most aspects of the $\Delta p p d$ mutant phenotype, but not the extended proliferation of stomatal lineage cells on hypocotyls, and leaves.

The expression of a set of genes selected from the RNA-Seq $\triangle p p d$ dataset as indicators of; stomatal development, light signalling, floral induction, procambium cell proliferation and hormone metabolism, was compared between $\triangle p p d$, phyBOE, and phyBOE;ppd (Fig. 6). For genes involved in light signalling (SPA1, AFR, ELF3) expression in the phyBOE and phyBOE;ppd genotypes was similar and opposite to the $\triangle p p d$ mutant. Expression of TOE2 was down-regulated in phyBOE, up-regulated in $\triangle p p d$ and intermediate between the two in the double mutant, whereas expression of NF-YB2 was down-regulated in all genotypes. Expression of $A C L 5$ was up-regulated in both phyBOE and phyBOE;ppd, in contrast to its down-regulation in the $\triangle p p d$ mutant. Unlike these examples, expression of $S P C H$ was upregulated in both $\triangle p p d$ and phyBOE;ppd but not in phyBOE, an expression pattern consistent with the meristemoid cell proliferation phenotypes (Supplementary Fig. S9).

Among the hormone biosynthesis genes examined (DWF4, TAA1, GA20ox3) expression in phyBOE and phyBOE;ppd was down-regulated, opposite to $\triangle p p d$ (Fig. 6b). Similarly, phyB overexpression altered transcription of the hormone signalling repressors, RGA1 and IAA14. Expression of the ABA activation gene, $B G 1$ was also similar in the phyBOE and phyBOE;ppd genotypes and opposite to the deletion mutant. In contrast to this epistatic effect of phyBOE on the expression of genes involved in $B R$, auxin, $G A$ and $A B A$ biosynthesis or activation, the expression of genes controlling hormone inactivation (BAT1, SUR2, GH3.5, CKX4) was similar in all the genotypes. Hence, phyBOE probably counteracted the ppd loss-of-function influence on BR, auxin and GA activities by altering the biosynthesis of these hormones. Expression of the cytokinin activation gene LOG5 was similar in $\triangle p p d$ and phyBOE;ppd, less in phyBOE seedlings but still up-regulated compared with wild type. Despite the possible increase in active $A B A$ due to up-regulation of $B G 1$ in phyBOE and phyBOE;ppd, the expression of ABA response genes bZIP1 and RD29A was similar in all genotypes. Collectively, results from genetic interactions between $C R Y 1 / P H Y B$ and $P P D$ and the pattern of gene expression in phyBOE;ppd plants, suggest the PPD genes 
act to modulate photomorphogenesis by regulating both the light signalling pathway and genes controlling hormone metabolism

Discussion. Increased leaf size resulting from reduced expression of the PPD1 and PPD2 genes in Arabidopsis has largely been attributed to the prolonged proliferation of meristemoids, precursor cells in the stomatal lineage. However, examination of PPD function in other species suggests there are alternative mechanisms for PPD control of organ size. In a recent report on the down-regulation of a $P P D$ ortholog in legumes, enhanced general cell division and not altered meristemoid cell proliferation, appears to have caused increases in leaf and seed size ${ }^{54}$. Here I report a more extensive phenotype for the Arabidopsis $\Delta p p d$ mutant that reveals a role for the $P P D$ genes as regulators of plasticity in growth and development throughout the life cycle. Features of the $\triangle p p d$ mutant phenotype include: tolerance to $\mathrm{ABA}$ inhibition of germination, hypocotyl elongation, increases in stomata number on hypocotyls, and (pro)cambium cell proliferation, delayed flowering time, and higher seed weight. Since the kix8;kix9 mutant phenocopies the $\Delta p p d$ mutant, the PPD interacting KIX proteins are also likely to be a part of the molecular mechanism regulating these diverse aspects of plant development.

Whole-genome analysis of transcription identified PPD target genes regulating light signalling and hormone homeostasis that provide a possible explanation for many aspects of the complex mutant phenotype. The PPD complex may act to either repress or activate the transcription of genes, and in some cases both modes of action seem to be used to regulate antagonistic components of a particular signalling or biosynthesis pathway. Examples include the repression of SPA1 and activation of AFR transcription to promote photomorphogenesis and the down-regulation of DWF4 and up-regulation of BAT1 to limit endogenous BR levels (Fig. 2b,c). Because light signalling influences many aspects of plant development by controlling endogenous phytohormone levels, it is possible that the PPD genes act only as positive regulators of the light signalling pathway. However, the $\Delta p p d$ mutant phenotype and its genetic interaction with cry1 and phyBOE suggest there are some aspects of PPD action that are independent of light signalling. Although there are many similarities between the shade avoidance response and the $\triangle p p d$ phenotype; $A B A$ insensitive seed germination, hypocotyl elongation, petiole elongation, down-regulation of anthocyanin biosynthesis and pathogen defence genes, and increased activity of the phytohormones, auxin, BR, GA, CK, other aspects of the mutant phenotype; delayed flowering, increased leaf and seed size and higher leaf stomatal density, are opposite to those expected from plants growing in shade. 
The PPD genes may also use a combination of direct and indirect (via light signalling) mechanisms to regulate specific developmental pathways. One example is the increased (pro)cambium proliferation of the $\Delta p p d$ mutant. Although the pathways controlling cambial cell proliferation are not fully understood it has been shown that the auxin dependent bHLH transcription factor TMO5 forms a heterodimer complex with another bHLH protein, LHW, and this TMO5-LHW complex directly up-regulates transcription of the CK biosynthesis genes LOG3 and LOG4, resulting in a CK mediated increase in (pro)cambium cell proliferation $^{55}$. Thermospermine produced by ACL5 represses the translational inhibitory effect of upstream ORFs located in the $5^{\prime}$ leader sequence of SACL genes. These genes encode bHLH transcription factors that compete with TMO5 for its LHW binding partner, thereby reducing the amount of active TMO5-LHW ${ }^{56-57}$. The PPD genes act as positive regulators of ACL5 transcription (Fig. 2b), and thereby may limit formation of the TMO5-LHW complex and cambial cell proliferation. Interestingly, light signalling also influences vascular growth. Loss-of-function mutants for phyB and hy5 have fewer xylem vessels in stem vascular than wild type ${ }^{58-59}$. This influence of the phytochromes on vascular growth may contribute to increased fitness in an exposed environment, since cucumber seedlings deficient in phyB grown outdoors exhibited low survival due to hypocotyl fracture ${ }^{58}$. Surprisingly then, defects in blue light, cryptochrome-mediated, signalling resulted in increased procambium cell proliferation in seedlings (Fig. 5). Since reduced cryptochrome signalling occurs in plants grown in shade, increased amounts of vascular tissue may be produced under these conditions to provide mechanical strength for elongating hypocotyls and petioles. The blocking of increased procambium cell proliferation in cry1 by PPDOE and in $\triangle p p d$ by phyBOE (Fig. $5 \mathrm{~d}$ ) suggest that a mutually antagonistic interaction between $P P D$ and light signalling modulates the rate of cambial cell proliferation. Because auxin activates TMO5 expression, and light signalling regulates the level of endogenous auxin, the effect of $P P D$ expression on cambial cell proliferation may result from a combination of direct control of ACL5 transcription and indirect regulation of auxin homeostasis via modulation of light signalling.

A mutually antagonistic interaction between $P P D$ and light signalling also regulates stomatal development on the hypocotyl (Fig. 5c). While it is known that stomata number on Arabidopsis hypocotyls is increased by BR or GA ${ }^{60-62}$ (Fig. 3), the influence of cryptochrome and phytochrome-mediated light signalling on hypocotyl stomatal development is unknown. Here I report that both cry1 and phyB act to limit stomata number on the hypocotyl. Overexpression of PPD1 blocked the increased stomata of a cry1 loss-of-function mutation, while phyB over-expression blocked the increase in stomata number on the hypocotyl of the $\triangle p p d$ mutant (Fig. 5c). The stomatal phenotype of phyBOE;ppd hypocotyls, which have 
extensive meristeomoid proliferation but no increase in stomata number, suggests excess activated phyB acts to inhibit stomatal development on hypocotyls downstream of PPD. Since over expression of phyB likely limits BR and GA hormone levels and $4 p p d$ deletion results in increased SPCH expression and a higher population of meristemoid cells in the epidermis, BR and GA maybe required for the development of the proliferating meristemoids into stomata. Collectively, these results suggest the intriguing possibility that seedlings respond to shading with a coordinate increase in hypocotyl length, stomata production and vascular growth, and that PPD provides a braking mechanism to prevent excessive developmental response to shading.

There are numerous possible explanations for the delayed flowering phenotype of the $\Delta p p d$ mutant. Expression of the flowering time gene $F T$ is mainly up-regulated in long days by the transcriptional activator $\mathrm{CO}$, and significantly, expression of some of the multiple regulatory components determining the activity of $\mathrm{CO}$ have been altered in the $\triangle p p d$ mutant. Examples include the up-regulation of TOE2 and the down-regulation of NF-YB2, both putative targets of PPD2 (Table II). TOE2 forms a complex with $\mathrm{CO}$ to suppress CO activation of $F T^{63}$, while NF-YB2 is a part of the NF-Y complex that enhances the binding of $\mathrm{CO}$ to the $F T$ promoter ${ }^{64}$. Other changes in the $\triangle p p d$ mutant that could lead to reduced CO activity were the increased expression of SPA1, since COP1-SPA1 complexes degrade $\mathrm{CO}^{65}$, and up-regulation of $R G A 1^{66}$. It is possible that a combination of these factors determine the delayed flowering of the $\triangle p p d$ mutant since expression of TOE2, SPA1 and RGA1 were reduced in PPDOE (Fig. 2). The down-regulation of NF-YB2 in $\triangle p p d$, phyBOE and phyB;ppd, together with phyBOE epistasis (Fig. 6), suggests that reduced expression of $N F-Y B 2$ did not significantly contribute to delayed flowering of the $\Delta p p d$ mutant.

Since both ppd and kix8;kix9 loss-of-function genotypes are insensitive to the inhibitory effect of ABA on seed germination (Fig. 3) the PPD complex maybe required for activation of the $A B A$ signalling pathway. Light signalling positively regulates $A B A$ response by $\mathrm{HY} 5$ binding to the promoter of $A B I 5$, a bZIP transcription factor controlling the expression of genes responsible for the inhibition of seed germination ${ }^{67}$. Although PPD might influence $A B A$ responses indirectly by modulating light signalling ${ }^{68}$, the results of gene expression profiling suggest an alternative molecular mechanism involving regulation of $b Z I P 1$, which encodes a transcription factor controlling many ABA response genes. Both $b Z I P 1$, and $R D 29 A$ which it activates, were down-regulated in $\triangle p p d$, phyBOE, and phyBOE;ppd (Fig. 6), all genotypes tolerant to the inhibitory effect of $A B A$ on seed germination (Fig. 5). This expression pattern may reflect a cascade effect, since $b Z I P 1$ is activated by the NF-Y 
complex, and NF-Y complex function is likely to be reduced in $\triangle p p d$, phyBOE and phyBOE;ppd due to the down-regulation of NF-Y2.

It has been reported that in Arabidopsis brassinosteroids modulate the transcription of genes for seed development specific pathways and are required for the development of normal seed size and weight ${ }^{69}$. Furthermore, overexpression of DWF4 results in increased seed weight ${ }^{51}$. Here I report increases in seed weight for $\Delta p p d$, kix8;kix9, and BR/1ox genotypes. In genetic interaction analysis PPDOE was epistatic to DWF4ox and BRI10x, whereas phyBOE was epistatic to $\triangle p p d$ (Figs. $4 \mathrm{~d} \& 5 \mathrm{~g}$ ). These results confirm a role for BR in the regulation of seed weight, and suggest that PPD limits growth of developing seeds by restricting $\mathrm{BR}$ biosynthesis, either directly or by modulating light signalling. Collectively, these results reveal a previously unrecognised role whereby PPD acts as a braking mechanism to limit developmental plasticity. Because PPD modulates developmental traits of economic importance there is potential to use tissue-specific alterations in PPD expression to obtain improvements in yield and stress tolerance in plants.

\section{Methods}

Plant Material. The Arabidopsis $\triangle p p d$ mutant and transgenic $\triangle p p d:: P P D 1$ complemented, and $\triangle p p d:: P P D 1$ over-expression (PPDOE) genotypes in a Landsberg erecta (Ler) ecotype background have been described previously ${ }^{38}$. All other Arabidopsis plants used in this study are in the Columbia ( $\mathrm{Col}-0$ ) background. The $\triangle p p d$ mutant and transgenic $\triangle p p d:: P P D O E$ genotypes were backcrossed with wild-type $\mathrm{Col}-\mathrm{O}$ for four generations to obtain $\triangle p p d \mathrm{Col}-\mathrm{O}$ and PPDOE Col-0. Mutants det2-1 (CS6159), cry1 (CS6955), and a transgenic phyB over expression genotype, phyBOE (35S::phyB, CS3081) were obtained from the Arabidopsis Biological Resource Center. Transgenic lines with over expression of DWF4 (35S::DWF4ox) or BRI1 (35S::BRI1ox) as previously described ${ }^{52}$, were supplied by Joanne Chory. The transgenic $P P D$ silencing line ami-ppd and the kix8;kix9 double mutant ${ }^{39}$, were supplied by Dirk Inzé. The det2, DWF4ox, BRI1ox, cry1, and phyBOE genotypes were crossed with $\triangle p p d$ or $P P D O E$ and double mutants (det2;ppd, cry1;ppd, phyBOE;ppd) or triple mutants (DWF4ox;ppd::PPDOE, BRI1ox;ppd::PPDOE, and cry1;ppd::PPDOE) selected from $\mathrm{F}_{2}$ populations. A combination of phenotype and genotyping by PCR with gene specific primers (Supplementary Table S2) identified homozygous lines.

Measurement of Flowering Time. Seeds were stratified at $4{ }^{\circ} \mathrm{C}$ for 3 days in the dark then germinated and plants grown in a soil mix in a controlled environment growth room at $22{ }^{\circ} \mathrm{C}$, $65 \%$ relative humidity, with $14 \mathrm{~h}$ white light/10 h dark cycles. Light intensity was $200 \mu \mathrm{m} \cdot \mathrm{m}^{-}$ ${ }^{2} \cdot \mathrm{s}^{-1}$ from a combination of Sylvania GRO-LUX F36W/GRO-T8 and Phillips TLD 58W/840 
fluorescent tubes. Flowering time was recorded as the number of days post germination taken for inflorescences to attained $1 \mathrm{~cm}$ in height. The experiment was replicated twice with 10 plants per genotype in each experiment.

Seed Weight Measurements. The weight of batches of 100 seeds of each genotype, were determined using a Mettler Toledo analytical balance. Results are the averages of seven replicates.

Seedling Growth Conditions. For hypocotyl growth, stomatal density, cotyledon vascular procambium cell proliferation, hormone response, RNA Seq and quantitative RT-PCR gene expression experiments, seed were surface sterilised with $70 \%$ ethanol, $0.01 \%$ Triton X-100 for $10 \mathrm{~min}$, followed by $100 \%$ ethanol for $5 \mathrm{~min}$, air dried on sterile filter paper, and transferred to media plates containing half-strength MS salts and vitamins (Duchefa Biochemie), $1 \%$ sucrose and $0.8 \%$ agar. Plates were then incubated for 3 days at $4{ }^{\circ} \mathrm{C}$ in the dark and grown at $24^{\circ} \mathrm{C}$ under a $14 \mathrm{~h}$ light $/ 10 \mathrm{~h}$ dark daily cycle. White light was provided by fluorescent tubes (Philips TLD 58W/840) at an intensity of $100 \mu \mathrm{m} \cdot \mathrm{m}^{-2} \cdot \mathrm{s}^{-1}$.

Microscopy. Hypocotyl length and hypocotyl stomatal density were scored on plate grown seedlings sampled 5 days post germination (dpg), cleared in 85\% lactic acid, for 1 min at $101^{\circ} \mathrm{C}, 15 \mathrm{psi}$, and mounted in the same solution on microscope slides. Total number of stomata on each hypocotyl was counted with an Olympus BX50 microscope using interference contrast $(n=40)$, while hypocotyl length was measured using a Leica Wild M3Z binocular microscope fitted with an eyepiece micrometer $(n=30)$. For observations on cotyledon stomatal development seedlings were sampled at $10 \mathrm{dpg}$ and cleared by passaging through the following solutions: $70 \% \mathrm{ETOH}, 70 \% \mathrm{ETOH} / 10 \%$ acetic acid, 1 ETOH:1 2.5M NaOH, each for $1 \mathrm{hr}$, then overnight in 85\% lactic acid. Whole plant images were obtained using an Olympus SZX12 binocular microscope and DP20 digital camera. Dark grown hypocotyl length was measured on seedlings sampled $4 \mathrm{dpg}$ after incubation in continuous darkness at $24^{\circ} \mathrm{C}$. For electron microscopy, hypocotyls sampled at $3 \mathrm{dpg}$ were observed directly using an FEI Quanta 200 environmental scanning electron microscope.

Histology. Plate grown seedlings sampled $10 \mathrm{dpg}$ were fixed in $4 \%$ glutaraldehyde, $1 \%$ formaldehyde, in $50 \mathrm{mM}$ phosphate buffer, $\mathrm{pH}$ 7, overnight, embedded in Technovit 7100 resin (Heraeus Kulzer) following the manufacturers protocol, and $7 \mu \mathrm{m}$ sections prepared using a Lieca RM2045 microtome. Sections were stained with Toluidine blue $O$, the number of procambium cells per cotyledon petiole vascular trace transverse section recorded $(n=30)$ and digital images obtained with an Olympus BX50 microscope analySIS ${ }^{\mathrm{B}}$ system. 
Transcript Analysis. Total RNA was extracted from ten-day-old seedlings grown on $0.5 \mathrm{MS}$ medium at mid-morning in the light cycle using an RNeasy Plant Kit (Qiagen) and genomic DNA contamination eliminated by on-column DNase I digestion using an RNase-Free DNase Set (Qiagen). RNA-Seq library preparation, high-throughput sequencing and data analysis services were provided by Oxford Gene Technology. RNA quality of the samples was determined using a 2100 Bioanalyzer (Agilent), and mRNA libraries prepared with the Illumina TruSeq RNA Sample Prep Kit v2. Sample sequencing was performed on an Illumina HiSeq2000 platform using TruSeq v3 chemistry. An average of 55,073,058 paired end reads was obtained per sample. Reads were mapped to the TAIR10 Arabidopsis genome assembly using Bowtie version 2.02, splice junctions identified using TopHat version 2.09 and Cufflinks version 2.1.1 was used to perform transcript assembly, abundance estimations and differential expression between wild-type and $\Delta p p d$ samples.

For real time reverse transcription quantitative PCR analysis first-strand complementary DNA was synthesised from $1 \mu \mathrm{g}$ total RNA, purified as described above, using both random hexamer and oligo(dT) $)_{18}$ primers and Maxima Reverse Transcriptase (Thermo Scientific) according to the manufacturer's protocol. Quantitative PCR analyses were undertaken using a LightCycler 480 real-time PCR instrument (Roche), LightCycler 480 SYBR Green Master reagents version 12 (Roche), and gene-specific oligonucleotide primers (Supplementary Table S3). Transcript abundance was calculated using LightCycler 480 instrument software based on the $\triangle \triangle C T$-method and relative expression of a gene was calculated from the ratio of test genotype samples to the reference Col-0 wild-type. Both actin2 and tubulin were used to normalise different samples. Four biological replicates and at least four technical replicates were used for each genotype.

Seedling Hormone-response Growth Assays. Epibrassinolide (Santa Cruz Biotechnology), and brassinazole (Santa Cruz Biotechnology) dissolved in dimethlsulphoxide (DMSO), gibberellic acid (ACROS organics) and picloram (Sigma) in ethanol, paclobutrazol (Sigma) in acetone, abscisic acid in methanol and 6-benzylaminopurine (Sigma) in $0.1 \mathrm{~N}$ $\mathrm{KOH}$, were filter sterilised and incorporated into 0.5 MS media plates. DMSO, ethanol, methanol or acetone as appropriate, were used for mock treatments. Seedlings were grown in light for 5 days before hypocotyl stomatal density $(n=30)$, hypocotyl length $(n=35)$ or root length $(n=15)$ at 4 days or percentage germination $(n=200)$ at 3 days post germination were analysed. Hypocotyl length $(n=20)$ of dark grown seedlings was analysed at 4 days post germination. Each treatment was repeated twice. 


\section{References}

1. Franklin, K.A., Quail, P.H. Phytochrome functions in Arabidopsis development. J. Exp. Bot. 61, 11-24 (2010)

2. Casal, J.J. Photoreceptor signaling networks in plant responses to shade. Annu. Rev. Plant Biol. 64, 403-427 (2013)

3. de Wit, M., Galvão, V.C., Fankhauser, C. Light-mediated hormonal regulation of plant growth and development. Annu. Rev. Plant Biol. 67, 513-537 (2016)

4. Tepperman, J.M. et al. Expression profiling of phyB mutant demonstrates substantial contribution of other phytochromes to red-light-regulated gene expression during seedling de-etiolation. Plant J. 38, 725-739 (2004)

5. Jiao, Y., Ma, L., Strickland, E., Deng, X.W. Conservation and divergence of lightregulated genome expression patterns during seedling development in rice and Arabidopsis. Plant Cell 17, 3239-3256 (2005)

6. Jiao, Y., Lau, O.S., Deng, X.W. Light-regulated transcriptional networks in higher plants. Nat. Rev. Genet. 8, 217-230 (2007)

7. Quail, P.H. Phytochrome photosensory signalling networks. Nat. Rev. Mol. Cell Biol. 3, 85-93 (2002)

8. Cluis, C.P., Mouchel, C.F., Hardtke, C.S. The Arabidopsis transcription factor HY5 integrates light and hormone signaling pathways. Plant J. 38, 332-347 (2004)

9. Lee, J. et al. Analysis of transcription factor HY5 genomic binding sites revealed its hierachical role in light regulation of development. Plant Cell 19, $731-749$ (2007)

10. Zhang, $\mathrm{H}$. et al. Genome-wide mapping of the HY5-mediated gene networks in Arabidopsis that involve both transcriptional and posttranscriptional regulation. Plant J. 65, 346-358 (2011)

11. Gangappa, S.N., Botto, J.F. The multifaceted roles of HY5 in plant growth and development. Mol. Plant 9, 1353-1365 (2016)

12. Saijo, Y. et al. The COP1-SPA1 interaction defines a critical step in phytochrome Amediated regulation of HY5 activity. Genes Dev. 17, 2642-2647 (2003)

13. Seo, H.S. et al. LAF1 ubiquitination by COP1 controls photomorphogenesis and is stimulated by SPA1. Nature 423, 995-999 (2003)

14. Lian, H.L. et al. Blue-light-dependent interaction of cryptochrome1 with SPA1 defines a dynamic signaling mechanism. Genes Dev. 25, 1023-1028 (2011)

15. Liu, B. et al. Arabidopsis cryptochrome1 interacts with SPA1 to suppress COP1 activity in response to blue light. Genes Dev. 25, 1029-1034 (2011) 
16. Lu, X-D. et al. Red-light-dependent interaction of phyB with SPA1 promotes COP1SPA1 dissociation and photomorphogenic development in Arabidopsis. Mol. Plant 8, 467-478 (2015)

17. Sheerin, D.J. et al. Light-activated phytochrome A and B interact with members of the SPA family to promote photomorphogenesis in Arabidopsis by reorganizing the COP1/SPA complex. Plant Cell 27, 189-201 (2015)

18. Lau, O.S., Deng, X.W. Plant hormone signaling lightens up: integrators of light and hormones. Curr. Opin. Plant Biol. 13, 571-577 (2010)

19. Barlier, I. et al. The SUR2 gene of Arabidopsis thaliana encodes the cytochrome P450 CYP83B1, a modulator of auxin homeostasis. Proc. Natl. Acad. Sci. USA 97, 14819-14824 (2000)

20. Tao, Y. et al. Rapid synthesis of auxin via a new tryptophan-dependent pathway is required for shade avoidance in plants. Cell 133, 164-176 (2008)

21. Halliday, K.J., Martínez-García, J.F., Josse, E-M. Integration of light and auxin signaling. Cold Spring Harb. Perspect. Biol. 2009;00:a001586

22. Neff, M.M. et al. BAS1: A gene regulating brassinosteroid levels and light responsiveness in Arabidopsis. Proc. Natl. Acad. Sci. USA 96, 15316-15323 (1999)

23. Poppenberger, B. et al. The UGT73C5 of Arabidopsis thaliana glycosylates brassinosteroids. Proc. Natl. Acad. Sci. USA 102, 15253-15258 (2005)

24. Tanaka, K. et al. Brassinosteroid homeostasis in Arabidopsis is ensured by feedback expressions of multiple genes involved in its metabolism. Plant Physiol. 138, 11171125 (2005)

25. Turk, E.M. et al. BAS1 and SOB7 act redundantly to modulate Arabidopsis photomorphogenesis via unique brassinosteroid inactivation mechanisms. Plant $\mathrm{J}$. 42, 23-34 (2005)

26. Kim, H.B. et al. The regulation of DWF4 expression is likely a critical mechanism in maintaining the homeostasis of bioactive brassinosteroids in Arabidopsis. Plant Physiol. 140, 548-557 (2006)

27. Achard, P. et al. DELLAs contribute to plant photomorphogenesis. Plant Physiol. 143, 1163-1172 (2007)

28. De Lucas, M. et al. A molecular framework for light and gibberellin control of cell elongation. Nature 451, 480-484 (2008)

29. Feng, S. et al. Coordinated regulation of Arabidopsis thaliana development by light and gibberellins. Nature 451, 475-479(2008)

30. Rieu, I. et al. The gibberellin biosynthetic genes AtGA200x1 and AtGA200x2 act, partially redundantly, to promote growth and development throughout the Arabidopsis life cycle. Plant J. 53, 488-504 (2008) 
31. Schneider, K. et al. Arabidopsis PIZZA has the capacity to acylate brassinosteroids. PloS ONE (10): e46805 (2012)

32. Choi, S. et al. BAT1, a putative acyltransferase, modulates brassinosteroid levels in Arabidopsis. Plant J. 73, 380-391 (2013)

33. Zhu, W. et al. Homeostasis of brassinosteroids regulated by DRL1, a putative acyltransferase in Arabidopsis. Mol. Plant 6, 546-558 (2013)

34. Shahnejat-Bushehri, S., Tarkowska, D., Sakuraba, Y., Balazadeh, S. Arabidopsis NAC transcription factor JUB1 regulates GA/BR metabolism and signalling. Nature Plants 2, 16013 (2016)

35. Donnelly, P.M., Bonetta, D., Tsukaya, H., Dengler, R.E., Dengler, N.G. Cell cycling and cell enlargement in developing leaves of Arabidopsis. Dev. Biol. 215, 407-419 (1999)

36. Bergmann, D.C., Sack, F.D. Stomatal development. Annu. Rev. Plant Biol. 58, 163181 (2007)

37. Geisler, M., Nadeau, J., Sack, F.D. Oriented asymmetric divisions that generate the stomatal spacing pattern in Arabidopsis are disrupted by the too many mouths mutation. Plant Cell 12, 2075-2086 (2000)

38. White, D.W.R. PEAPOD regulates lamina size and curvature in Arabidopsis. Proc. Natl. Acad. Sci. USA 103, 13238-13243 (2006)

39. Gonzalez, N. et al. A repressor protein complex regulates leaf growth in Arabidopsis. Plant Cell 27, 2273-2287 (2015)

40. Pauwels, L., Goossens, A. The JAZ proteins: a crucial interface in the jasmonate signalling cascade. Plant Cell 23, 3089-3100 (2011)

41. Pauwels, L. et al. NINJA connects the co-repressor TOPLESS to jasmonate signaling. Nature 464, 788-791 (2010)

42. Wang, Z. et al. SCF ${ }^{\mathrm{SAP}}$ controls organ size by targeting PPD proteins for degradation in Arabidopsis thaliana. Nature Commun. 7, 11192 (2016)

43. MacAlister, C.A., Ohashi-Ito, K., Bergmann, D.C. Transcription factor control of asymmetric cell divisions that establish the stomatal lineage. Nature 445, 537-540 (2007)

44. Lau, O.S. et al. Direct roles of SPEECHLESS in the specification of stomatal selfrenewing cells. Science 345, 1605-1609 (2014)

45. Harmon, F.G., Kay, S.A. The F box protein AFR is a positive regulator of phytochrome A-mediated light signaling. Curr. Biol. 13, 2091-2096 (2003)

46. Zhou, P. et al. Both PHYTOCHROME RAPIDLY REGULATED1 (PAR1) and PAR2 promote seedling photomorphogenesis in multiple light signaling pathways. Plant Physiol. 164, 841-852 (2014) 
47. Li, L. et al. PIL1 participates in a negative feedback loop that regulates its own gene expression in response to shade. Mol. Plant 7, 1582-1585 (2014)

48. Hartmann, L. et al. Crosstalk between two bZIP signaling pathways orchestrates saltinduced metabolic reprogramming in Arabidopsis roots. Plant Cell 27, 2244-2260 (2015)

49. Ursache, R., Nieminen, K., Helariutta, Y. Genetic and hormonal regulation of cambial development. Physiol. Plant. 147, 36-45 (2013)

50. Clay, N.K., Nelson, T. Arabidopsis thickvein mutation affects vein thickness and organ vascularization, and resides in a provascular cell-specific spermine synthase involved in vein definition and in polar auxin transport. Plant Physiol. 138, 767-777 (2005)

51. Choe, S. et al. Overexpression of DWF4 in the brassinosteroid biosynthetic pathway results in increased vegetative growth and seed yield in Arabidopsis. Plant J. 26, 573-582 (2001)

52. Wang, Z-Y., Seto, H., Fujioka, S., Yoshida, S., Chory, J. BRI1 is a critical component of a plasma-membrane receptor for plant steroids. Nature 410, 380-383 (2001)

53. Chory, J., Nagpal, P., Peto, C.A. Phenotypic and genetic analysis of det2, a new mutant that affects light-regulated seedling development in Arabidopsis. Plant Cell 3, 445-459 (1991)

54. Liangfa, G. et al. Increasing seed size and quality by manipulating BIG SEEDS1 in legume species. Proc. Natl. Acad. Sci. 113, 12414-12419 (2016)

55. De Rybel, B. et al. Integration of growth and patterning during vascular tissue formation in Arabidopsis. Science 345, 1255215 (2014)

56. Katayama, H. et al. A negative feedback loop controlling bHLH complexes is involved in vascular cell division and differentiation in the root apical meristem. Curr. Biol. 25, 3144-3150 (2015)

57. Vera-Sirera, F. et al. A bHLH-based feedback loop restricts vascular cell proliferation in plants. Dev. Cell 35, 432-443 (2015)

58. Casal, J.J., Ballaré, C.L., Tourn, M., Sánchez, R.A. Anatomy, growth and survival of a long-hypocotyl mutant of Cucumis sativus deficient in phytochrome B. Ann. Bot. 73, 569-575 (1994)

59. Oyama, T., Shimura, Y., Okada, K. The Arabidopsis HY5 gene encodes a bZIP protein that regulates stimulus-induced development of root and hypocotyl. Genes Dev. 11, 2983-2995 (1997)

60. Fuentes, S., Cañamero, R.C., Serna, L. Relationship between brassinosteroids and genes controlling stomatal production in the Arabidopsis hypocotyl. Int. J. Dev. Biol. $56,675-680(2012)$ 
61. Gudesblat, G. E. et al. SPEECHLESS integrates brassinosteroid and stomata signalling pathways. Nat. Cell Biol. 14, 548-554 (2012)

62. Saibo, N.J.M., Vriezen, W.H., Beemster, G.T.S., Vandef Straeten, D. Growth and stomata development of Arabidopsis hypocotyls are controlled by gibberellins and modulated by ethylene and auxins. Plant J. 33, 989-1000 (2003)

63. Zhang, B., Wang, L., Zeng, L., Zhang, C., Ma, H. Arabidopsis TOE proteins convey a photoperoidic signal to antagonize CONSTANS and regulate flowering time. Genes Dev. 29, 975-987 (2015)

64. Cao, S. et al. A distal CCAAT/NUCLEAR FACTOR Y complex promotes chromatin looping at the FLOWERING LOCUS T promoter and regulates the timing of flowering in Arabidopsis. Plant Cell 26, 1009-1017 (2014)

65. Zuo, Z., Liu, H., Liu, B., Liu, X., Lin, C. Blue light-mediated interaction of CRY2 with SPA1 regulates COP1 activity and floral initiation in Arabidopsis. Curr. Biol. 21, 841847 (2011)

66. Xu, F. et al. DELLA proteins physically interact with CONSTANS to regulate flowering under long days in Arabidopsis. FEBS Letters 590, 541-549 (2016)

67. $\mathrm{Xu}, \mathrm{D}$. et al. Convergence of light and ABA signaling on the ABI5 promoter. PloS Genet. 10, e1004197 (2014)

68. Chen, H. et al. Integration of light and abscisic acid signaling during seed germination and early seedling development. Proc. Natl. Acad. Sci. USA 105, 4495-4500 (2008)

69. Jiang, W-B. et al. Brassinosteroid regulates seed size and shape in Arabidopsis. Plant Physiol. 162, 1965-1977 (2013)

\section{Acknowledgements.}

This work was funded by grants from Royal Society of New Zealand Marsden Fund (AGR304), Foundation for Research, Science \& Technology (C10X0816) and Cambium Genetics. I thank the Manawatu Microscopy \& Imaging Centre for the use of microscopy resources.

Competing financial interests: The author declares no competing financial interests. 


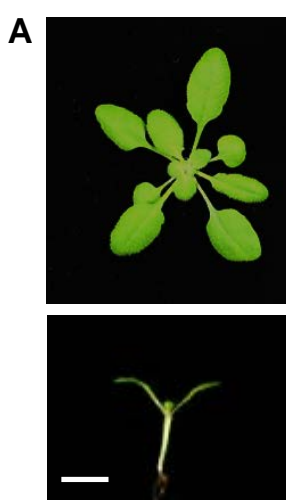

Col-0

B

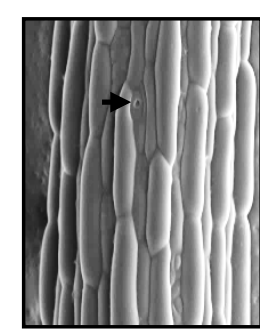

Col-0

C

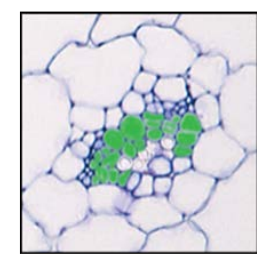

Col-0

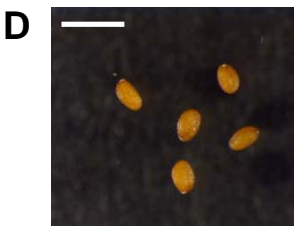

Col-0

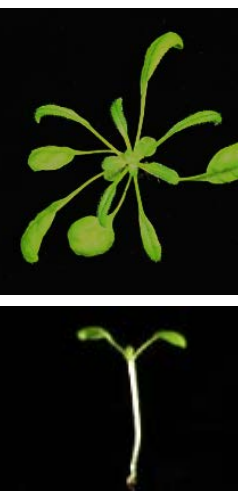

$\Delta p p d$

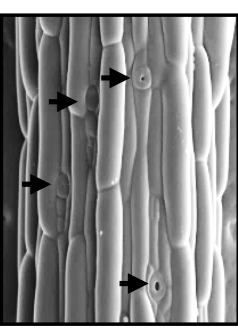

$\Delta p p d$

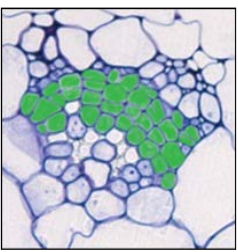

$\triangle p p d$

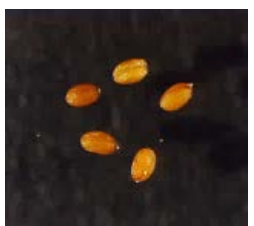

$\Delta p p d$

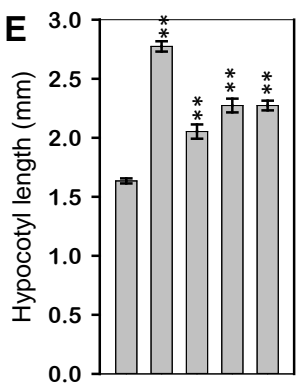

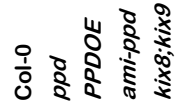
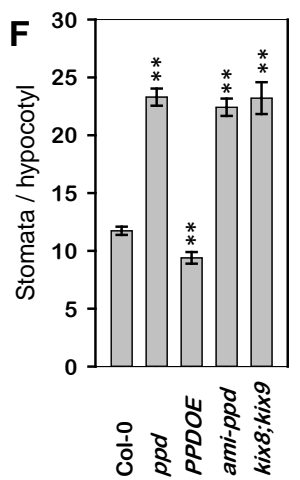

G

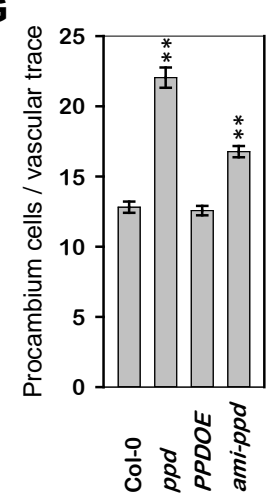

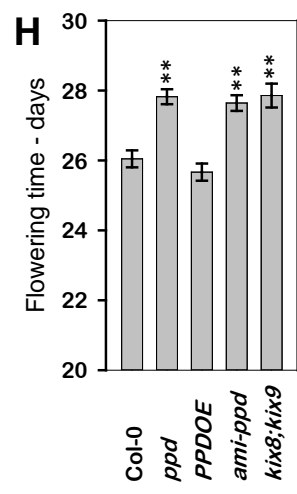

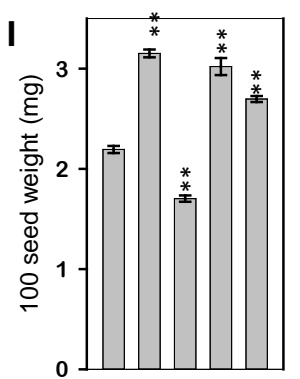

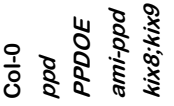

Figure 1. PPD has a multifaceted role in plant growth and development. Comparison of the phenotypes of wild type Col-0, $\triangle p p d$ deletion, and PPDOE over expression genotypes, (A) mature plants at 21 days post

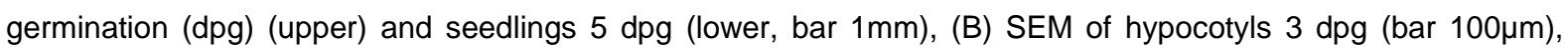
black arrows indicate stomata, (C) transverse sections of cotyledon petiole vascular traces at $10 \mathrm{dpg}$, with procambium cells shaded green, and (D) representative examples of seed size (bar $1 \mathrm{~mm}$ ). Graphs compare wild type, $\triangle p p d$, PPDOE, ami-ppd, and kix8;kix9 genotypes for (E) hypocotyl length $5 \mathrm{dpg},(\mathrm{n}=30)$, (F) stomata number per hypocotyl $5 \mathrm{dpg}$, (n=40), (G) 100 seed weight, $(n=7)$ and $(H)$ days to flowering for plants grown in a $14 \mathrm{hr}$ light:10 hr dark photoperiod, $(\mathrm{n}=20)$. (I) Comparison of procambium cell proliferation in the cotyledon petiole vascular traces of wild type, $\triangle p p d, P P D O E$ and ami-ppd seedlings at $10 \mathrm{dpg},(\mathrm{n}=30) .{ }^{\star \star} P<0.001$ compared with the wild type (2-tailed Student's $t$-test). Error bars represent $\pm \mathrm{SE}$. 
A

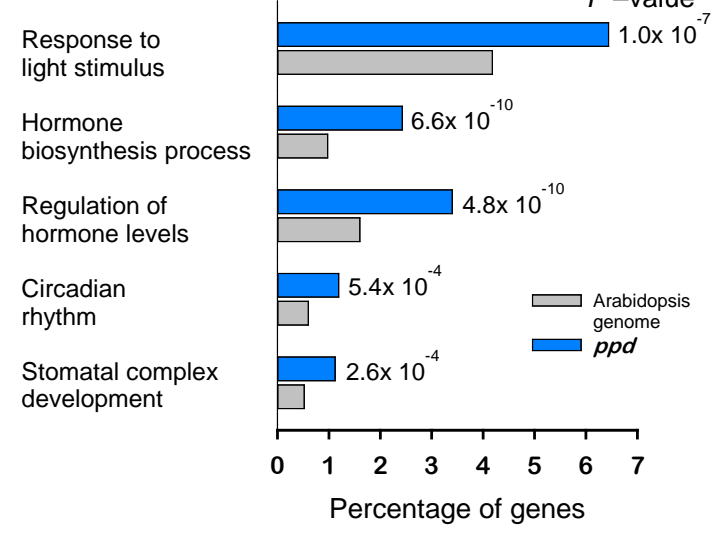

C

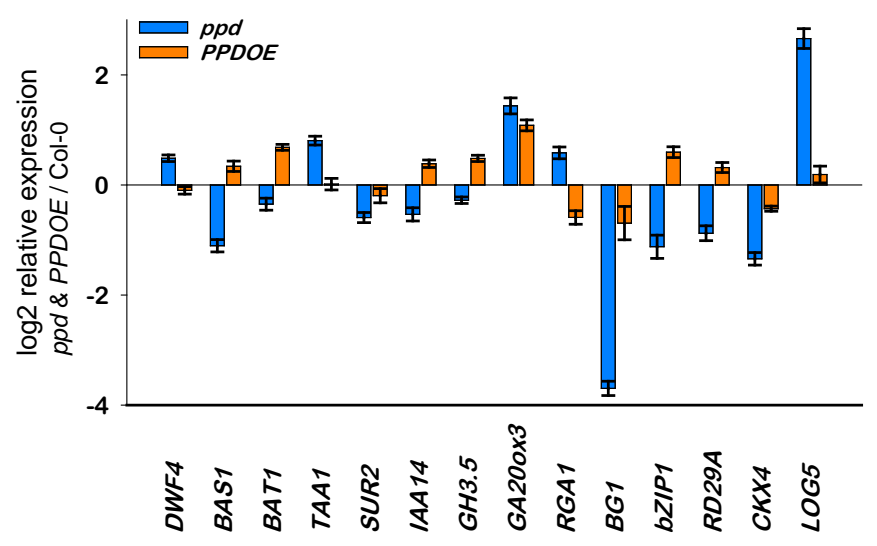

B

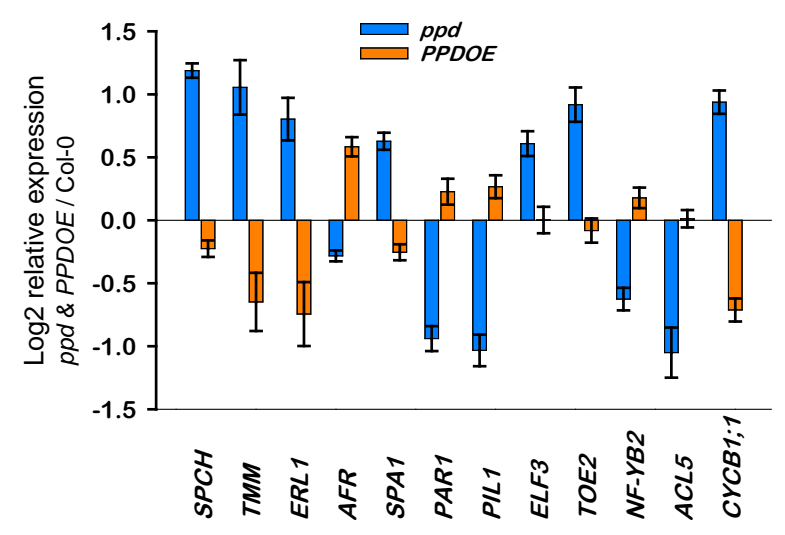

D

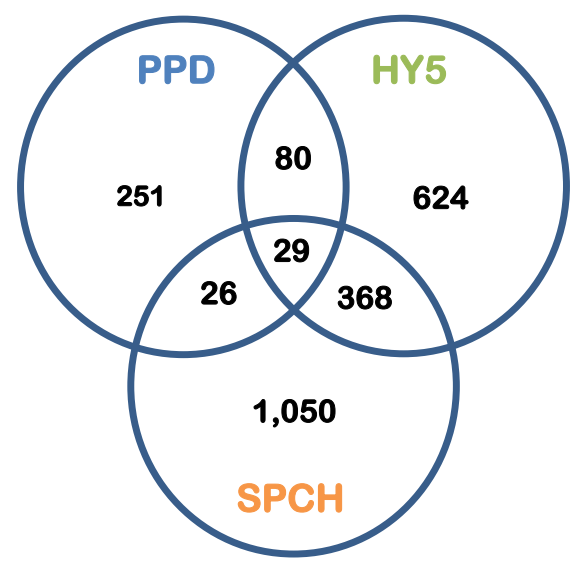

Figure 2. Genome-wide differential gene expression between wild type and $\triangle p p d$ mutant reveals PPD has roles regulating light signalling, circadian rhythm, hormone metabolism and stomatal development. (A) A selected subset of the Gene Ontology terms significantly enriched among the genes identified by RNA-Seq analysis of seedlings as differential expressed in $\Delta p p d$. RT-qPCR expression analysis of selected genes regulating (B) stomatal development, light signalling, circadian rhythm, photoperiodic flowering, and cambium proliferation, and (C) hormone biosynthesis, catabolism, conjugation and signalling genes, compared the $\Delta p p d$ mutant and PPDOE over expression genotypes relative to wild type. (D) Diagram of the number of genes differentially expressed in the $\triangle p p d$ mutant that are putative binding targets of PPD2, SPCH or HY5. Error bars represent $\pm \mathrm{SE}$ 

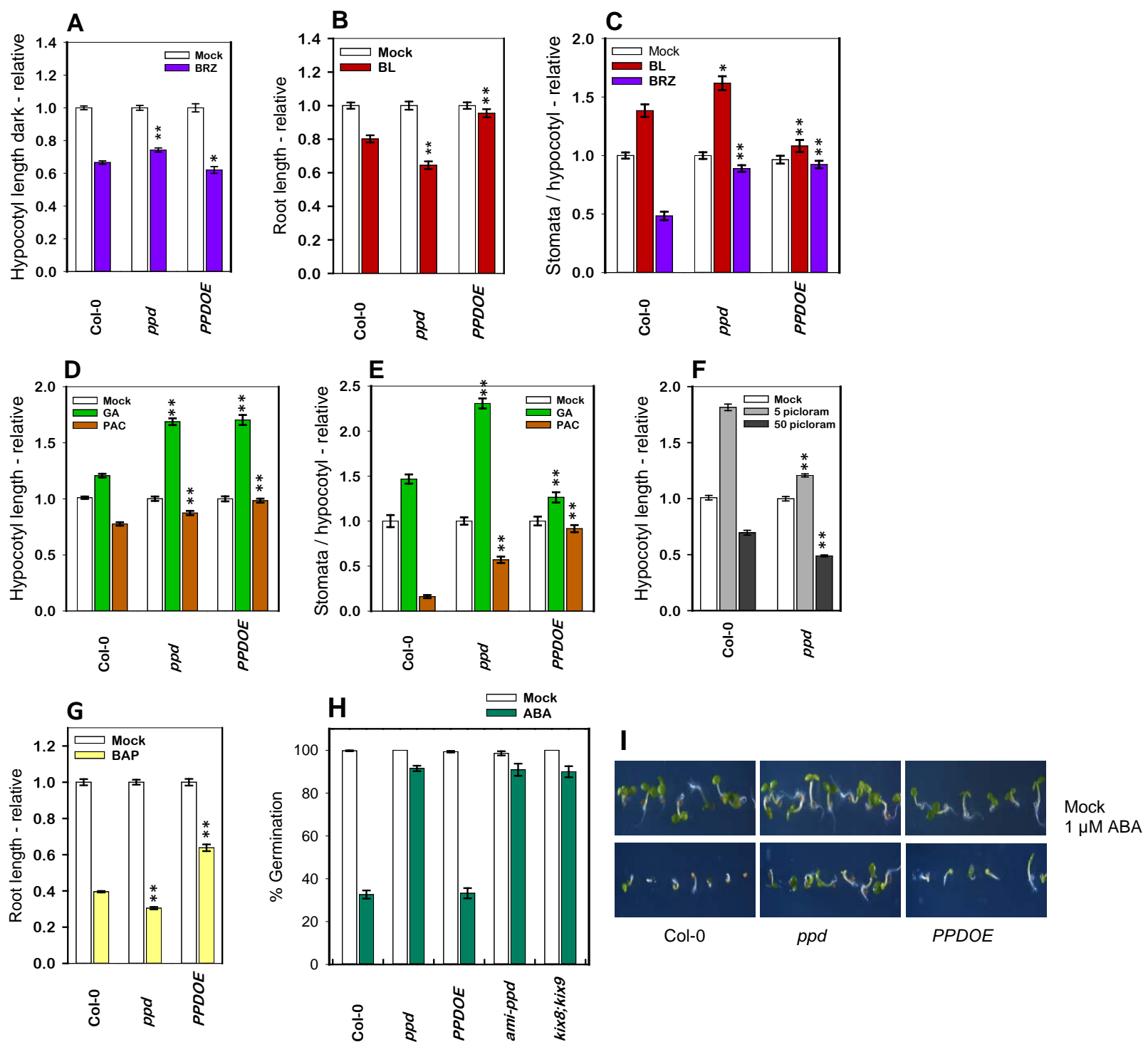

Figure 3. PPD modulates growth and developmental responses to hormones and inhibitors of hormone biosynthesis. A-F Responses to hormone or hormone biosynthesis inhibitor treatments graphed as relative to

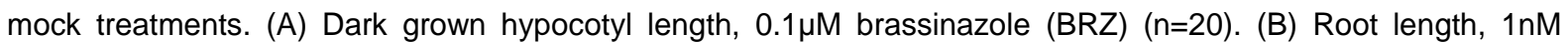

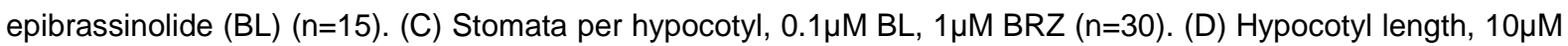

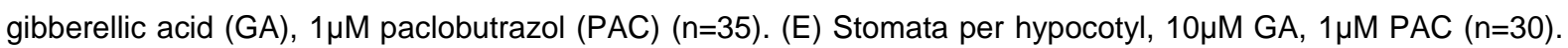

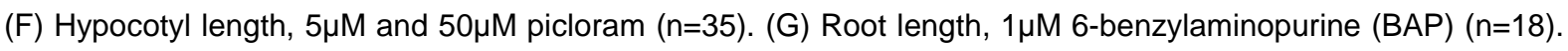
(H) Percentage seed germination at 3 days post stratification, $1 \mu \mathrm{M}$ abscisic acid (ABA). (I) Photographs taken at $4 \mathrm{dpg}$ illustrating genotype differences in germination and greening in response to treatment with $1 \mu \mathrm{M} A B A$. Significant differences from wild type ${ }^{*} P<0.05$ or ${ }^{*} P<0.001$ (2-tailed Student's $t$-test). Error bars represent \pm SE. 
A

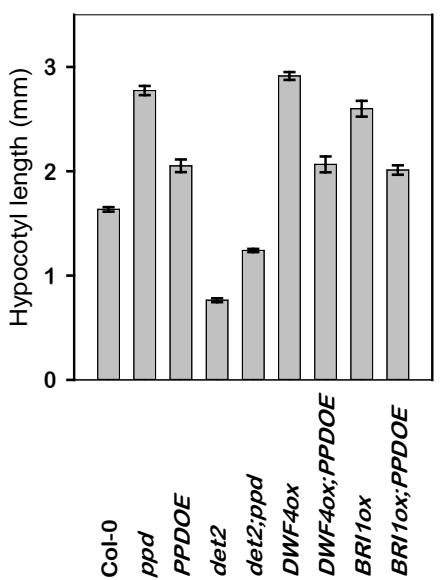

D

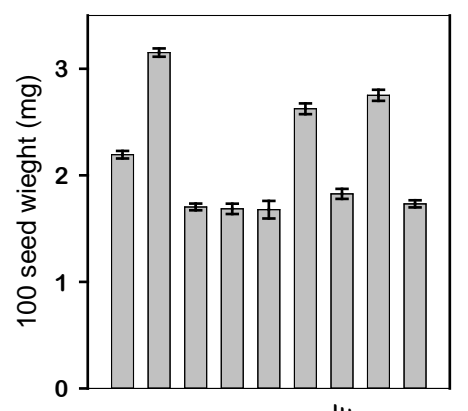

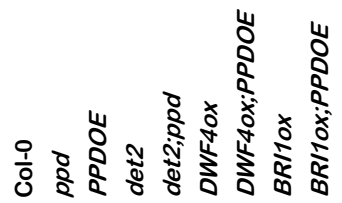

B

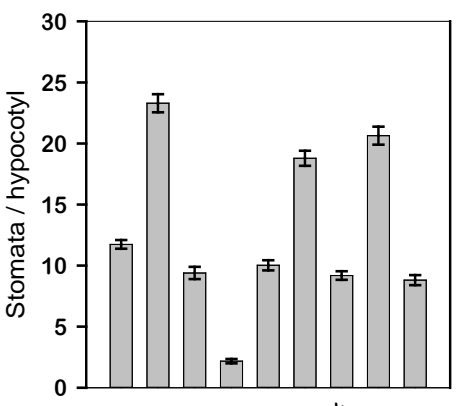

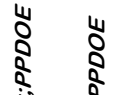

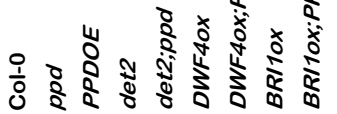

E

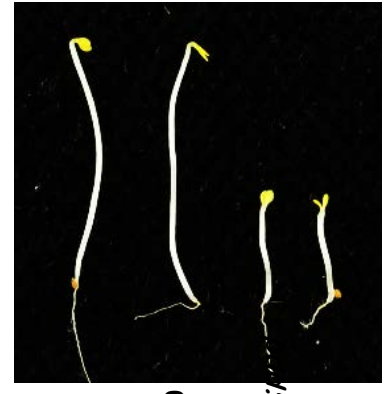

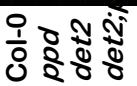

C

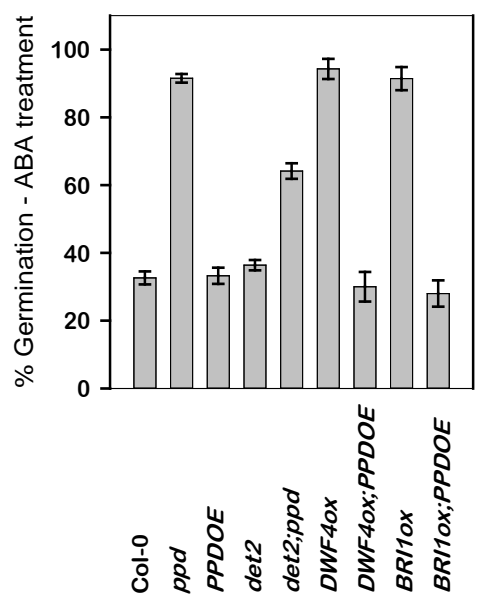

$\mathbf{F}$

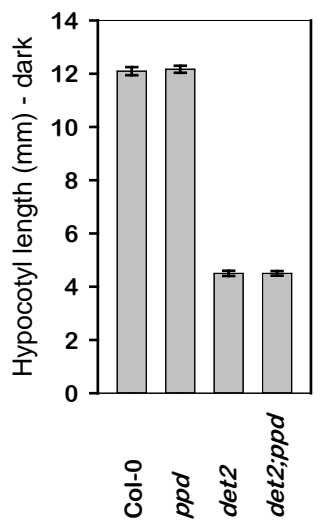

Figure 4. $P P D$ is a negative regulator of BR biosynthesis. Genetic analysis of interactions between genotypes with altered $P P D$ function $(\triangle p p d, P P D O E)$ and genotypes with altered BR metabolism or signal transduction (det2, reduced biosynthesis, DWF4ox, increased biosynthesis, and BRI1ox, increased BR signalling). (A) Hypocotyl length, $(n=30)$. (B) Stomata per hypocotyl, $(n=40)$. (C) Percentage germination in the presence of $1 \mu M$ ABA. (D) Seed weight. (E) Photograph of dark grown seedlings 4 dpg. (F) Hypocotyl length of dark grown seedlings $(n=20)$. Error bars represent \pm SE. 
A

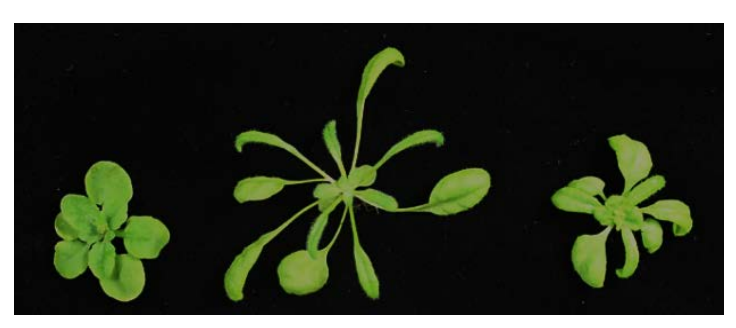

PhyBOE

$\Delta p p d$

D

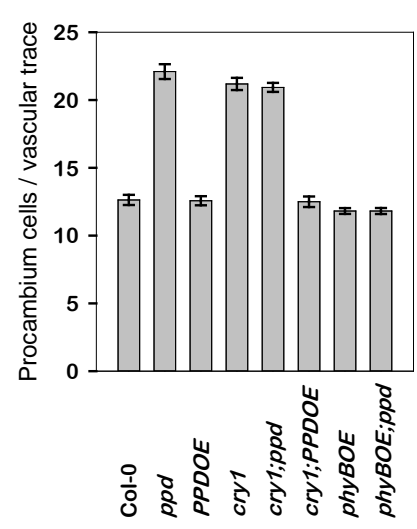

B
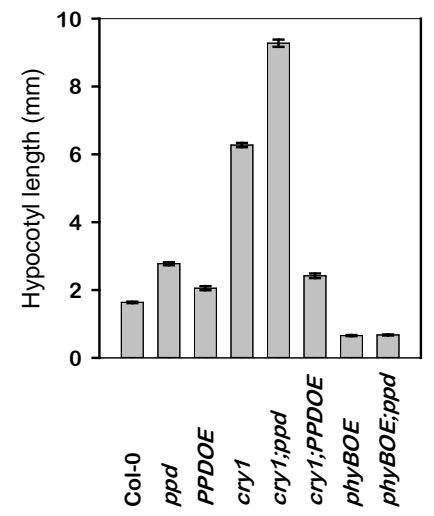

E

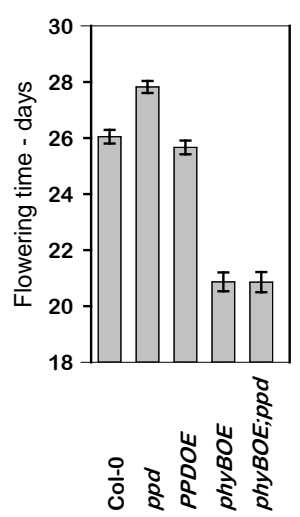

C

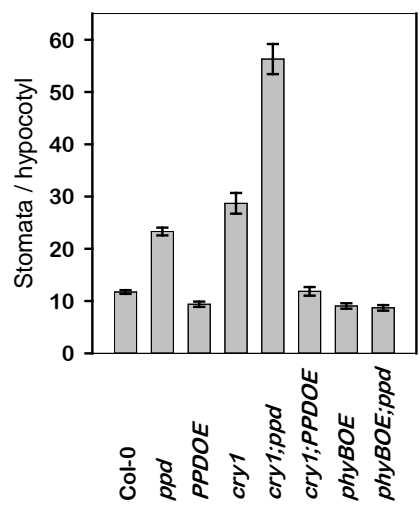

G

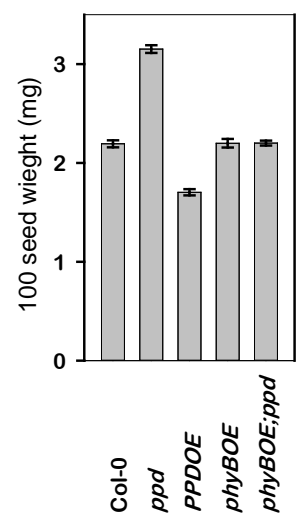

Figure 5. PPD genetically interacts with $C R Y 1$ and $P H Y B$ to regulate light signalling. (A) Comparison of phyBOE (over expression of phytochrome B), $\triangle p p d$, and $\triangle p p d$;phyBOE 21 dpg. (B) Hypocotyl length. (C) Stomata per hypocotyl. (D) Procambium cells per vascular trace section. (E) Flowering time. (F) Germination sensitivity to ABA. (G) Seed weight. Error bars represent \pm SE. 

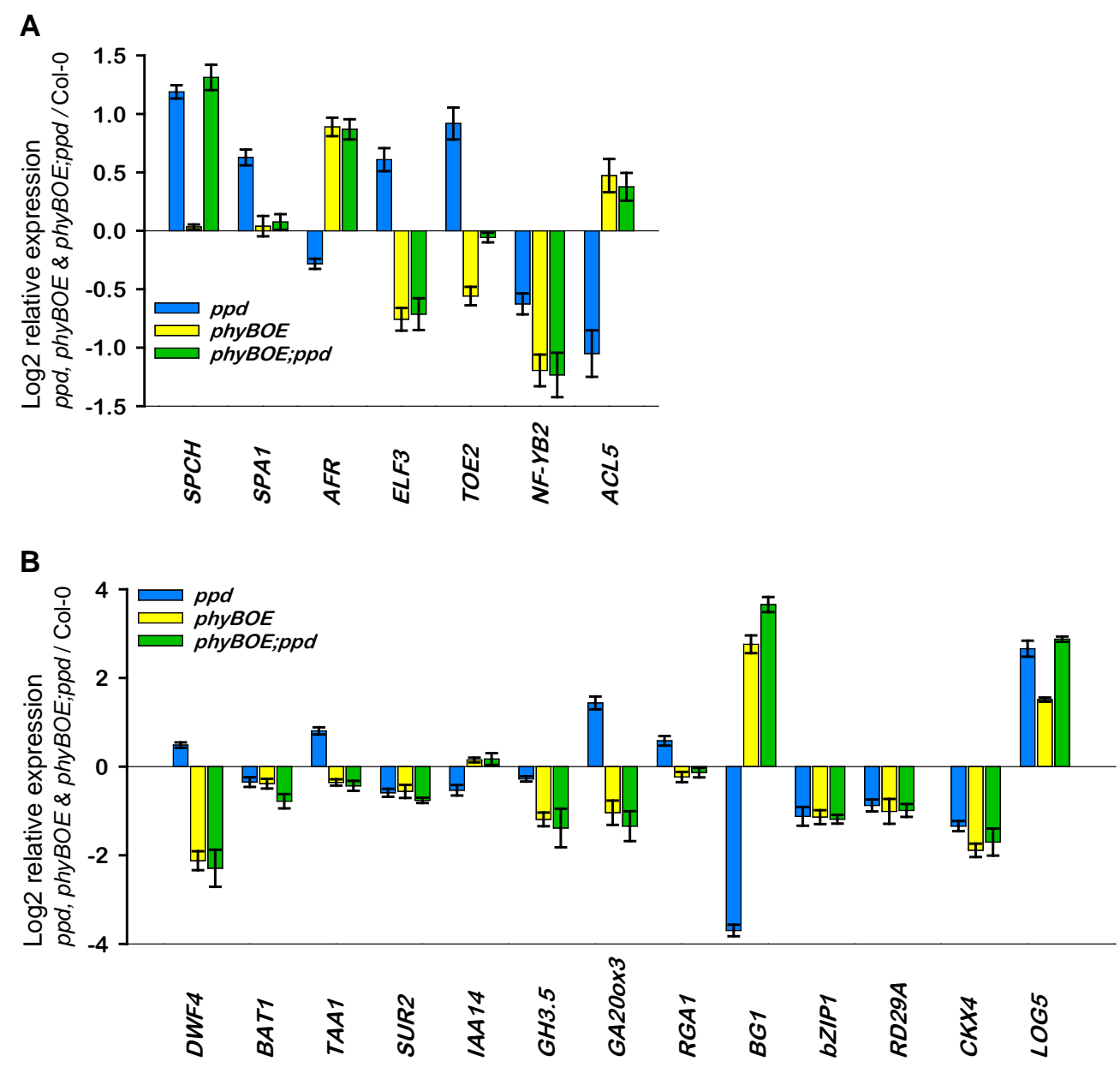

Figure 6. $P H Y B$ over expression alters the pattern of $\triangle p p d$ gene expression. RT-qPCR expression analysis of selected genes regulating (A) stomatal development, light signalling, circadian rhythm, photoperiodic flowering, and cambium proliferation, and (B) hormone biosynthesis, catabolism, conjugation and signalling genes. Error bars represent \pm SE. 
bioRxiv preprint doi: https://doi.org/10.1101/102707; this version posted January 23, 2017. The copyright holder for this preprint (which was not certified by peer review) is the author/funder. All rights reserved. No reuse allowed without permission.

Table I. Selected genes differentially expressed in $\triangle p p d$. Putative PPD2 targets are in bold.

\begin{tabular}{|c|c|c|c|c|}
\hline AGI Code & Gene Symbol & $\log _{2}$ Fold Change & $P$ value & Gene function \\
\hline \multicolumn{5}{|c|}{ (A) Stomatal development } \\
\hline AT1G05230 & HDG2 & 0.5078 & 0.0016 & HD-ZIP IV protein, promotes stomatal development \\
\hline AT1G12860 & SCRM2 & 0.5127 & 0.0008 & bHLH TF, determinant of progression of stomatal cell lineage \\
\hline AT1G26600 & CLE9 & 0.8623 & 0.00225 & CLE peptide, possible role in meristemoid maintenance \\
\hline AT1G34245 & EPF2 & 1.1897 & 0.00005 & Secretory peptide, controls asymmetric cell division in stomatal lineage \\
\hline AT1G80080 & $T M M$ & 0.9809 & 0.00005 & Receptor, promotes asymmetric cell division in stomata development \\
\hline AT3G26744 & SCRM1 & 0.6699 & 0.00005 & bHLH TF, determinant of progression of stomatal cell lineage \\
\hline AT3G57230 & AGL16 & 0.4426 & 0.0055 & MADS box TF, promotes stomata from satellite meristemoids \\
\hline AT5G07180 & ERL2 & 1.1209 & 0.00005 & Receptor-like kinase, maintains stomatal stem cell activity \\
\hline AT5G53210 & $\mathrm{SPCH}$ & 0.8734 & 0.00005 & bHLH TF, establishes stomatal cell lineage \\
\hline AT5G60880 & $B A S L$ & 1.3827 & 0.00005 & Regulator of asymmetrically dividing stomatal-lineage cells \\
\hline AT5G62230 & ERL1 & 1.1739 & 0.00005 & Receptor-like kinase, maintains stomatal stem cell activity \\
\hline
\end{tabular}

(B) Light signalling, circadian rhythm, and flowering genes.

(i) Light signalling

$\begin{array}{lllll}\text { AT2G24540 } & \text { AFR } & -0.6003 & 0.0001 & \text { F-box protein positive regulator of phyA signalling } \\ \text { AT2G42870 } & \text { PAR1 } & -0.6727 & 0.0028 & \text { bHLH TF, negative regulator of shade avoidance } \\ \text { AT2G46340 } & \text { SPA1 } & 0.4398 & 0.0049 & \text { Regulator of phyA signalling, repressor of photomorphogenesis } \\ \text { AT2G46970 } & \text { PIL1 } & -1.8117 & 0.00005 & \text { Regulates light-mediated inhibition of hypocotyl elongation } \\ \text { (ii) } \underline{\text { Circadian rhythm }} & & & & \\ \text { AT1G22770 } & \text { GI } & 0.5658 & 0.0004 & \text { Regulates circadian clock phyB signalling and flowering time } \\ \text { AT2G25930 } & \text { ELF3 } & 0.5306 & 0.0017 & \text { Circadian clock component, interacts with phyB to control development } \\ \text { AT5G24470 } & \text { PRR5 } & 1.4340 & 0.00005 & \text { Regulates circadian clock components and photomorphogenesis } \\ \text { AT5G59570 } & \text { BOA } & 1.2499 & 0.00075 & \text { Component of circadian clock }\end{array}$

(iii) Flowering

$\begin{array}{lllll}\text { AT5G47640 } & \text { NF-YB2 } & -0.7127 & 0.0019 & \text { Interacts with CO, required for transcription of } F T \\ \text { AT5G60120 } & \text { TOE2 } & 0.4797 & 0.00165 & \text { AP2 TF, represses FT expression }\end{array}$

(C) Hormone biosynthesis, catabolism, conjugation and signalling genes.

(i) Abscisic acid

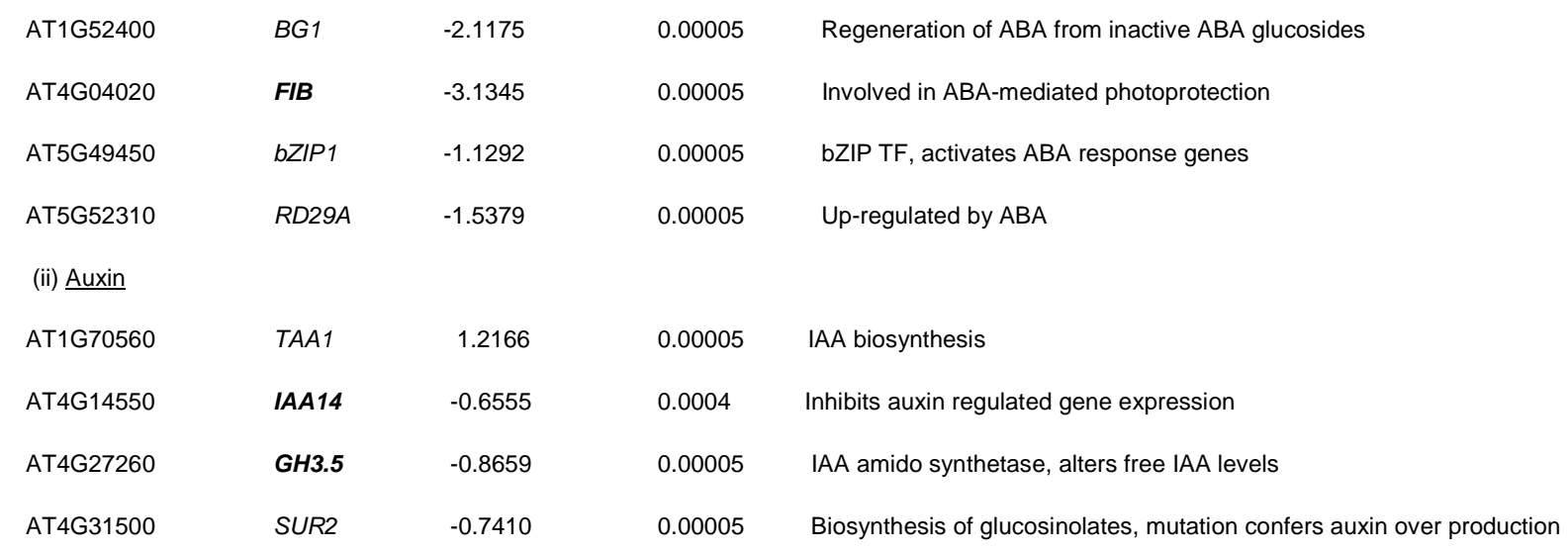


bioRxiv preprint doi: https://doi.org/10.1101/102707; this version posted January 23, 2017. The copyright holder for this preprint (which was not certified by peer review) is the author/funder. All rights reserved. No reuse allowed without permission.

(iii) Brassinosteroid

\begin{tabular}{|c|c|c|c|c|}
\hline AT2G26710 & BAS1 & -1.5351 & 0.00005 & Cytochrome P450, inactivates BRs \\
\hline AT2G43000 & JUB1 & -2.8141 & 0.0001 & NAC TF, negative regulator of DWF4 \\
\hline AT3G50660 & DWF4 & 0.6257 & 0.00005 & $22 \alpha$ hydrolase, rate limiting step in BR biosynthetic pathway \\
\hline $\begin{array}{l}\text { AT4G31910 } \\
\text { (iv) Cytokinin }\end{array}$ & BAT1 & -0.5246 & 0.0048 & Acylation of BRs, may modulate BR levels \\
\hline AT4G29740 & $C K X 4$ & -2.5910 & 0.00005 & Degradation of cytokinins \\
\hline $\begin{array}{l}\text { AT4G35190 } \\
\text { (v) Gibberelli }\end{array}$ & LOG5 & 1.2124 & 0.00075 & Cytokinin activation \\
\hline AT2G01570 & RGA1 & 0.5187 & 0.0006 & Transcriptional regulator repressing GA response \\
\hline AT5G07200 & GA20ox3 & 1.5074 & 0.00655 & GA biosynthesis \\
\hline
\end{tabular}

$\mathrm{TF}$, transcription factor 